\title{
UNIQUENESS OF SOLUTION OF A HETEROGENEOUS EVOLUTION DAM PROBLEM ASSOCIATED WITH A COMPRESSIBLE FLUID FLOW THROUGH A RECTANGULAR POROUS MEDIUM
}

\author{
Elmehdi ZaOuche \\ University of EL Oued, Algeria
}

\begin{abstract}
This paper is concerned with the uniqueness of a weak solution of an evolution dam problem arising in a compressible fluid flow through a two-dimensional, rectangular, and heterogeneous porous medium. Our problem is associated with the equation $a\left(x_{1}\right)\left(u_{x_{2}}+\chi\right)_{x_{2}}-$ $(u+\chi)_{t}=0$. The technique we use is based on a transformation of the weak form of this equation into a similar one that enables us to argue as in [12].
\end{abstract}

\section{INTRODUCTION}

In this paper, we consider the following weak formulation of the evolution dam problem with heterogeneous coefficients which arises in the flow of a compressible fluid:

(1.1)

$$
\left\{\begin{array}{l}
\text { Find }(u, \chi) \in L^{2}\left(0, T ; H^{1}(\Omega)\right) \times L^{\infty}(Q) \text { such that: } \\
u \geq 0, \quad 0 \leq \chi \leq 1, \quad u .(1-\chi)=0 \text { a.e. in } Q, \quad u=\phi \text { on } \Sigma_{2}, \\
\int_{Q}\left[a\left(x_{1}\right)\left(u_{x_{2}}+\chi\right) \xi_{x_{2}}-(u+\chi) \xi_{t}\right] d x d t \leq \int_{\Omega}\left(\chi_{0}(x)+u_{0}(x)\right) \xi(x, 0) d x, \\
\forall \xi \in H^{1}(Q), \xi=0 \text { on } \Sigma_{3}, \xi \geq 0 \text { on } \Sigma_{4}, \xi(x, T)=0 \text { for a.e. } x \in \Omega,
\end{array}\right.
$$

where $\Omega=(0, L) \times(0, H)$ is a bounded rectangular domain in $\mathbb{R}^{2}$; the generic point in $\Omega$ is denoted by $x=\left(x_{1}, x_{2}\right)$ and represents a porous medium with

2020 Mathematics Subject Classification. 35A02, 35B35, 76S05.

Key words and phrases. Heterogeneous evolution dam problem, compressible fluid flow, rectangular porous medium, uniqueness. 
a boundary $\partial \Omega=\Gamma_{1} \cup \Gamma_{2}$ such that $\Gamma_{1}=[0, L] \times\{0\}, \Gamma_{2}=(\{0\} \times[0, H]) \cup$ $([0, L] \times\{H\}) \cup(\{L\} \times[0, H]) . Q=\Omega \times(0, T), T>0$ is a positive real number, $\phi$ is a nonnegative Lipschitz continuous function defined in $\bar{Q}, \Sigma_{1}=$ $\Gamma_{1} \times(0, T), \Sigma_{2}=\Gamma_{2} \times(0, T), \Sigma_{3}=\Sigma_{2} \cap\{\phi>0\}$, and $\Sigma_{4}=\Sigma_{2} \cap\{\phi=0\}$. Note that from a physical point of view, $\Gamma_{1}$ is the impervious part of $\partial \Omega, \Gamma_{2}$ is the part in contact with either air or fluid reservoirs, and $\phi$ stands for the assigned pressure on $\Sigma_{2}$. For some constants $0<\lambda \leq \Lambda, a\left(x_{1}\right)$ is a function of the variable $x_{1}$ that satisfies

$$
\lambda \leq a\left(x_{1}\right) \leq \Lambda \quad \text { a.e. } x_{1} \in(0, L)
$$

and $u_{0}, \chi_{0}: \Omega \rightarrow \mathbb{R}$ are functions satisfying for some positive constant $M$,

$$
\begin{aligned}
& 0 \leq \chi_{0}(x) \leq 1 \quad \text { a.e. } x \in \Omega, \\
& 0 \leq u_{0}(x) \leq M \quad \text { a.e. } x \in \Omega .
\end{aligned}
$$

The strong formulation of (1.1) associated with the initial data $\left(u_{0}, \chi_{0}\right)$ is given by

$$
\left\{\begin{aligned}
u \geq 0,0 \leq \chi \leq 1, u(1-\chi) & =0 & & \text { in } Q, \\
a\left(x_{1}\right)\left(u_{x_{2}}+\chi\right)_{x_{2}}-(u+\chi)_{t} & =0 & & \text { in } Q, \\
u & =\phi & & \text { on } \Sigma_{2}, \\
u(\cdot, 0)+\chi(\cdot, 0) & =u_{0}+\chi_{0} & & \text { in } \Omega, \\
a\left(x_{1}\right)\left(u_{x_{2}}+\chi\right) \cdot \nu & =0 & & \text { on } \Sigma_{1}, \\
a\left(x_{1}\right)\left(u_{x_{2}}+\chi\right) \cdot \nu & \leq 0 & & \text { on } \Sigma_{4},
\end{aligned}\right.
$$

where $\nu$ denotes the outward unit normal to $\partial \Omega$.

Concerning the question of existence of a solution to problem (1.1), we refer to [2], where an existence theorem was established for a class of nonstationary free boundary problems. Also, a regularity in time result for this class was obtained in [9].

Uniqueness of the solution was proved in [3] for a homogeneous medium in the general geometry by using the method of doubling variables. For a heterogeneous rectangular dam, the uniqueness was obtained in [12] in the case of an incompressible flow. By a different method, uniqueness was obtained in [5] and [10] for a rectangular dam wet at the bottom and dry near to the top, respectively, the homogeneous and heterogeneous cases, in both compressible and incompressible fluids. We also refer to [1], for the uniqueness of the solution of the evolution free boundary problem in theory of lubrication.

In this article, we prove the uniqueness of the solution for problem (1.1) by transforming the weak form of the equation $a\left(x_{1}\right)\left(u_{x_{2}}+\chi\right)_{x_{2}}-(u+\chi)_{t}=0$ in $Q$ into a form that does not contain the time term in $u$ (see Proposition 2.2) which allows us to apply the proof of uniqueness in the incompressible case (see [12]). We can also establish some properties of the solutions as in [12, 
Sect. 2], though it is difficult to adapt the proof of the uniqueness obtained in [12] with the same properties.

\section{UNIQUENESS OF THE SOLUTION}

We assume throughout this section that

$$
a \in C^{1}([0, L]) .
$$

First, we have the following regularity result of solutions of problem (1.1) ([9]).

Proposition 2.1. We have

$$
\begin{array}{ll}
\chi \in C^{0}\left([0, T] ; L^{p}(\Omega)\right), & \forall p \in[1,+\infty), \\
u \in C^{0}\left([0, T] ; L^{p}(\Omega)\right), & \forall p \in[1,2] .
\end{array}
$$

The following proposition plays a key role in the proof of the uniqueness result.

Proposition 2.2. Let $(u, \chi)$ be a solution of problem (1.1). Then

$$
\forall \xi \in H_{0}^{1}(\Omega), \forall t \in[0, T]: \quad \int_{\Omega} a\left(x_{1}\right)\left(u_{x_{2}}+\chi\right) \xi_{x_{2}} d x=0 .
$$

Proof. Without loss of generality, assume that $\xi \in \mathcal{D}(\Omega)$. Then we obtain the result by approximation for $\xi \in H_{0}^{1}(\Omega)$. Let $\kappa$ be a fixed element in $(0, T]$. We define the following function $\eta$ on $[0, T]$ by

$$
\eta(t)= \begin{cases}2\left(\frac{t}{\delta}\right)^{2} & \text { if } t \in\left[0, \frac{\delta}{2}\right] \\ 1-2\left(1-\frac{t}{\delta}\right)^{2} & \text { if } t \in\left(\frac{\delta}{2}, \delta\right] \\ 1 & \text { if } t \in(\delta, \kappa-\delta] \\ 1-2\left(1-\frac{\kappa-t}{\delta}\right)^{2} & \text { if } t \in\left(\kappa-\delta, \kappa-\frac{\delta}{2}\right] \\ 2\left(\frac{\kappa-t}{\delta}\right)^{2} & \text { if } t \in\left(\kappa-\frac{\delta}{2}, \kappa\right] \\ 0 & \text { if } t \in(\kappa, T]\end{cases}
$$

where $\delta$ is a positive real number. We can see that $\eta \in C^{1}([0, T]), \eta(0)=$ $\eta(T)=0$ and

$$
\eta^{\prime}(t)=\left\{\begin{array}{ll}
4 \frac{t}{\delta^{2}} & \text { if } t \in\left[0, \frac{\delta}{2}\right] \\
\frac{\delta}{\delta}\left(1-\frac{t}{\delta}\right) & \text { if } t \in\left(\frac{\delta}{2}, \delta\right] \\
0 & \text { if } t \in(\delta, \kappa-\delta] \\
-\frac{4}{\delta}\left(1-\frac{\kappa-t}{\delta}\right) & \text { if } t \in\left(\kappa-\delta, \kappa-\frac{\delta}{2}\right] \\
-\frac{4}{\delta}\left(\frac{\kappa-t}{\delta}\right) & \text { if } t \in\left(\kappa-\frac{\delta}{2}, \kappa\right] \\
0 & \text { if } t \in(\kappa, T]
\end{array} .\right.
$$


For all $\xi \in \mathcal{D}(\Omega)$, the function $\xi \eta^{2}$ belongs to $C^{1}(Q)$ and satisfies $\xi \eta^{2}=0$ on $\partial \Omega \times(0, T)$ and $\left(\xi \eta^{2}\right)(., 0)=\left(\xi \eta^{2}\right)(., T)=0$ in $\Omega$. Therefore, $\pm \xi \eta^{2}$ are test functions for (1.1) and we have

$$
\begin{aligned}
0 & =\int_{\Omega \times(0, \kappa)}\left[a\left(x_{1}\right)\left(u_{x_{2}}+\chi\right) \xi_{x_{2}} \eta^{2} d x d t-\int_{\Omega \times(0, \kappa)} 2(u+\chi) \eta \eta^{\prime} \xi d x d t\right. \\
& =: I_{\delta}^{1}-I_{\delta}^{2} .
\end{aligned}
$$

Applying the Lebesgue dominated convergence theorem to $I_{\delta}^{1}$, we obtain

$$
\lim _{\delta \rightarrow 0} I_{\delta}^{1}=\int_{\Omega \times(0, \kappa)} a\left(x_{1}\right)\left(u_{x_{2}}+\chi\right) \xi_{x_{2}} d x d t .
$$

Moreover, we can use the definition of $\eta^{\prime}$ to observe that the quantity $\left|I_{\delta}^{2}\right|$ can be estimated as follows:

$$
\begin{aligned}
\left|I_{\delta}^{2}\right| & =2\left|\int_{\Omega} \int_{0}^{\delta}(u+\chi) \eta \eta^{\prime} \xi d x d t+\int_{\Omega} \int_{\kappa-\delta}^{\kappa}(u+\chi) \eta \eta^{\prime} \xi d x d t\right| \\
& \leq 2 C\left\{\int_{0}^{\delta}|u+\chi|_{1, \Omega} \eta\left|\eta^{\prime}\right| d t+\int_{\kappa-\delta}^{\kappa}|u+\chi|_{1, \Omega} \eta\left|\eta^{\prime}\right| d t\right\} \\
& =: 2 C\left(I_{\delta}^{2,1}+I_{\delta}^{2,2}\right),
\end{aligned}
$$

where $C=\sup _{x \in \Omega}|\xi(x)|$. Let $f(t)=|u+\chi|_{1, \Omega} \eta$. Notice that $f$ is rightcontinuous and vanishes at 0 since $u, \chi \in C^{0}\left([0, T] ; L^{1}(\Omega)\right.$ ) (see Proposition 2.1), $\eta \in C^{0}([0, \kappa])$ and $\eta(0)=0$. So, by using $\left|\eta^{\prime}\right| \sim \frac{1}{\delta}$, we get

$$
\lim _{\delta \rightarrow 0} I_{\delta}^{2,1}=0 .
$$

Similarly, the function $f$ is left-continuous and vanishes at $\kappa$ and $\left|\eta^{\prime}\right| \sim \frac{1}{\delta}$; then

$$
\lim _{\delta \rightarrow 0} I_{\delta}^{2,2}=0 .
$$

Hence, by letting $\delta \rightarrow 0$ in (2.4) and using (2.5)-(2.6), we get

$$
\lim _{\delta \rightarrow 0} I_{\delta}^{2}=0 .
$$

Using (2.3) and (2.7) we obtain by taking $\delta \rightarrow 0$ in (2.2):

$$
\forall \kappa \in(0, T]: \quad \int_{\Omega \times(0, \kappa)} a\left(x_{1}\right)\left(u_{x_{2}}+\chi\right) \xi_{x_{2}} d x d t=0
$$

which leads, using integration by parts, to

$$
\forall \kappa \in(0, T]: \quad 0=\int_{0}^{\kappa} \int_{\Omega} a\left(x_{1}\right)\left(\chi \xi_{x_{2}}-u \xi_{x_{2} x_{2}}\right) d x d t:=F(\kappa) .
$$

Hence

$$
\forall t \in[0, T]: \quad F^{\prime}(t)=\int_{\Omega} a\left(x_{1}\right)\left(\chi \xi_{x_{2}}-u \xi_{x_{2} x_{2}}\right) d x=0
$$


since $t \mapsto \int_{\Omega} a\left(x_{1}\right)\left(\chi \xi_{x_{2}}-u \xi_{x_{2} x_{2}}\right) d x$ is continuous on $[0, T]$. Performing again an integration by parts, we obtain (2.1).

The uniqueness theorem is now presented.

TheOREM 2.3. Let $\left(u_{1}, \chi_{1}\right)$, and $\left(u_{2}, \chi_{2}\right)$ be two solutions of problem (1.1) such that $u_{1}(., 0)+\chi_{1}(., 0)=u_{2}(., 0)+\chi_{2}(., 0)=u_{0}+\chi_{0}$ a.e. in $\Omega$. Then

$$
\left(u_{1}, \chi_{1}\right)=\left(u_{2}, \chi_{2}\right) \text { a.e. in } Q \text {. }
$$

We first need to prove the following proposition.

Proposition 2.4. If $\left(u_{1}, \chi_{1}\right)$ and $\left(u_{2}, \chi_{2}\right)$ are two solutions of (1.1), we have

$$
\begin{aligned}
& \int_{Q} a\left(x_{1}\right)\left\{\left(u_{1}(x, t)-u_{2}(x, t)\right)_{x_{2}}^{+}+\left(1-\chi_{2}(x, t)\right) \chi_{\left\{u_{1}>u_{2}\right\}}\right\} \eta \xi_{x_{2}} d x d t \leq 0, \\
& \forall \xi \in \mathcal{D}(\Omega), \quad \xi \geq 0, \quad \forall \eta \in \mathcal{D}(0, T), \quad \eta \geq 0 .
\end{aligned}
$$

Proof. Let $\xi \in \mathcal{D}(\Omega)$ and $\eta \in \mathcal{D}(0, T)$ such that $\xi \geq 0$ and $\eta \geq 0$. For a positive real number $\delta$, consider the following functions $\rho_{1, \delta}(r)=\frac{1}{\delta} \rho_{1}\left(\frac{r}{\delta}\right)$, $\rho_{2, \delta}(r)=\frac{1}{\delta} \rho_{2}\left(\frac{r}{\delta}\right), \rho_{3, \delta}(r)=\frac{1}{\delta} \rho_{3}\left(\frac{r}{\delta}\right)$ with $\rho_{1}, \rho_{2}, \rho_{3} \in \mathcal{D}(\mathbb{R}), \rho_{1}, \rho_{2}, \rho_{3} \geq 0$, $\operatorname{supp}\left(\rho_{1}\right), \operatorname{supp}\left(\rho_{2}\right), \operatorname{supp}\left(\rho_{3}\right) \subset(-1,1)$. Let for all $(x, t, y, s) \in \overline{Q \times Q}$,

$$
\begin{aligned}
\zeta(x, t, y, s)= & \xi\left(\frac{x_{1}+y_{1}}{2}, \frac{x_{2}+y_{2}}{2}\right) \eta\left(\frac{t+s}{2}\right) \rho_{1, \delta}\left(\frac{x_{1}-y_{1}}{2}\right) \\
& \times \rho_{2, \delta}\left(\frac{x_{2}-y_{2}}{2}\right) \rho_{3, \delta}\left(\frac{t-s}{2}\right) .
\end{aligned}
$$

By choosing $\delta$ small enough, we obtain

$$
\begin{array}{ll}
\forall(t, y, s) \in(0, T) \times Q: & \zeta(\cdot, t, y, s)=0 \quad \text { on } \partial \Omega, \\
\forall(x, t, s) \in Q \times(0, T): \quad \zeta(x, t, \cdot, s)=0 & \text { on } \partial \Omega .
\end{array}
$$

With this notation of doubling variables, we consider that the solution $\left(u_{1}, \chi_{1}\right)$ has the variable $(x, t)$ and $\left(u_{2}, \chi_{2}\right)$ has the variable $(y, s)$. So, for a positive real number $\epsilon$, we set

$$
\vartheta(x, t, y, s)=\min \left(\frac{\left(u_{1}(x, t)-u_{2}(y, s)\right)^{+}}{\epsilon}, \zeta(x, t, y, s)\right) .
$$

From (2.9), the function $\xi()=.\vartheta(., t, y, s) \in H_{0}^{1}(\Omega)$ for a.e. $(t, y, s) \in(0, T) \times$ $Q$. Therefore, we can apply Proposition 2.2 to $\left(u_{1}, \chi_{1}\right)$ with $\xi()=.\vartheta(., t, y, s)$ to get

$$
\int_{\Omega} a\left(x_{1}\right)\left(u_{1 x_{2}}+\chi_{1}\right) \vartheta_{x_{2}} d x=0 .
$$

By integrating over $(0, T) \times Q$, we obtain

$$
\int_{Q \times Q} a\left(x_{1}\right)\left(u_{1 x_{2}}+\chi_{1}\right) \vartheta_{x_{2}} d x d t d y d s=0 .
$$


E. ZAOUCHE

In addition, if we use $u_{1}\left(1-\chi_{1}\right)=0$ a.e. in $Q$, one can easily verify that for a.e. $(x, t, y, s) \in Q \times Q$ :

$$
\chi_{1} a\left(x_{1}\right)\left(\min \left(\frac{\left(u_{1}-u_{2}\right)^{+}}{\epsilon}, \zeta\right)\right)_{x_{2}}=a\left(x_{1}\right)\left(\min \left(\frac{\left(u_{1}-u_{2}\right)^{+}}{\epsilon}, \zeta\right)\right)_{x_{2}} .
$$

Therefore, (2.11) takes the form

$$
\int_{Q \times Q} a\left(x_{1}\right)\left(u_{1 x_{2}}+1\right) \vartheta_{x_{2}} d x d t d y d s=0 .
$$

Similarly, with (2.10), we can see that for all $(x, t, s) \in Q \times(0, T)$, applying Proposition 2.2 to $\left(u_{2}, \chi_{2}\right)$ with $\xi()=.\vartheta(x, t, ., s)$, we get

$$
\int_{\Omega} a\left(y_{1}\right)\left(u_{2 y_{2}}+\chi_{2}\right) \vartheta_{y_{2}} d y=0 \text {. }
$$

Integrating over $Q \times(0, T)$, we find

$$
\int_{Q \times Q} a\left(y_{1}\right)\left(u_{2 y_{2}}+\chi_{2}\right) \vartheta_{y_{2}} d x d t d y d s=0 .
$$

By subtracting (2.13) from (2.12), we obtain

$\int_{Q \times Q}\left[a\left(x_{1}\right) u_{1 x_{2}} \vartheta_{x_{2}}-a\left(y_{1}\right) u_{2 y_{2}} \vartheta_{y_{2}}+a\left(x_{1}\right) \vartheta_{x_{2}}-\chi_{2} a\left(y_{1}\right) \vartheta_{y_{2}}\right] d x d t d y d s=0$.

To derive (2.8), we continue the same argument as in the proof of Theorem 3.1 in [12] (after the relation (3.9)) with the use of (2.14) and the method of doubling variables.

Proof of Theorem 2.3. If $\left(u_{1}, \chi_{1}\right)$ and $\left(u_{2}, \chi_{2}\right)$ are two solutions of (1.1), let

$$
v=\left(u_{1}-u_{2}\right)^{+} \text {and } \gamma=\left(1-\chi_{2}\right) \chi_{\left\{u_{1}>u_{2}\right\}} .
$$

Insert $v$ and $\gamma$ in $(2.8)$ yields

$$
\int_{Q} \eta a\left(x_{1}\right)\left(v_{x_{2}}+\gamma\right) \xi_{x_{2}} d x d t \leq 0, \forall \xi \in \mathcal{D}(\Omega), \xi \geq 0, \eta \in \mathcal{D}(0, T), \eta \geq 0 .
$$

Using (2.15) and applying a similar argument to the first part of the proof of Theorem 3.4 of [12] (after the relation (3.38)), we get

$$
u_{1}=u_{2}:=u \quad \text { a.e. in } Q .
$$

Moreover, let $\xi \in \mathcal{D}(\Omega)$ and let $\eta$ be the function defined in Proposition 2.2. For both $\left(u, \chi_{1}\right)$ and $\left(u, \chi_{1}\right)$, we can choose $\pm \xi \eta^{2}$ as test functions in (1.1) to obtain that for all $\kappa \in(0, T]$,

$$
\begin{aligned}
& \int_{\Omega \times(0, \kappa)}\left\{a\left(x_{1}\right)\left(u_{x_{2}}+\chi_{1}\right) \xi_{x_{2}} \eta^{2}-2\left(u+\chi_{1}\right) \eta \eta^{\prime} \xi\right\} d x d t=0, \\
& \int_{\Omega \times(0, \kappa)}\left\{a\left(x_{1}\right)\left(u_{x_{2}}+\chi_{2}\right) \xi_{x_{2}} \eta^{2}-2\left(u+\chi_{2}\right) \eta \eta^{\prime} \xi\right\} d x d t=0 .
\end{aligned}
$$


By subtracting (2.18) from (2.17) and using (2.16), we obtain

$$
\int_{\Omega \times(0, \kappa)} a\left(x_{1}\right)\left(\chi_{1}-\chi_{2}\right) \xi_{x_{2}} \eta^{2} d x d t-\int_{\Omega \times(0, \kappa)} 2\left(\chi_{1}-\chi_{2}\right) \eta \eta^{\prime} \xi d x d t=0
$$

Finally, using (2.19), we obtain by an argument similar to that in the second part of the proof of [12, Theorem 3.4],

$$
\chi_{1}=\chi_{2} \quad \text { a.e. in } Q,
$$

which completes the proof of Theorem 2.3.

\section{REFERENCES}

[1] S. J. Alvarez and R. Oujja, On the uniqueness of the solution of an evolution free boundary problem in theory of lubrication, Nonlinear Anal. 54 (2003), 845-872.

[2] M. Bousselsal, A. Lyaghfouri and E. Zaouche, On the existence of a solution of a class of non-stationary free boundary problems, Glas. Mat. Ser. III 53(73) (2018), 449-475.

[3] J. Carrillo, On the uniqueness of the solution of the evolution dam problem, Nonlinear Anal. 22 (1994), 573-607.

[4] J. Carrillo and G. Gilardi, La vitesse de propagation dans le problème de la digue, Ann. Fac. Sci. Toulouse Math. (5) 11 (1990), 7-28.

[5] E. DiBenedetto and A. Friedam, Periodic behaviour for the evolutionary dam problem and related free boundary problems, Comm. Partial Differential Equations 11 (1986), 1297-1377.

[6] G. Gilardi, A new approach to evolution free boundary problems, Comm. Partial Differential Equations 4 (1979), 1099-1123; 5 (1980), 983-984.

[7] D. Gilbarg and N. S. Trudinger, Elliptic partial differential equations of second order, Springer, New York, 1983.

[8] A. Lyaghfouri, The evolution dam problem for nonlinear Darcy's law and Dirichlet boundary conditions, Portugal. Math. 56 (1999), 1-37.

[9] A. Lyaghfouri and E. Zaouche, $L^{p}$-continuity of solutions to parabolic free boundary problems, Electron. J. Differ. Equations 2015, No. 184, 9 pp.

[10] A. Lyaghfouri and E. Zaouche, Uniqueness of solution of the unsteady filtration problem in heterogeneous porous media, Rev. R. Acad. Cienc. Exactas Fis. Nat. Ser. A Math. RACSAM 112 (2018), 89-102.

[11] A. Torelli, Existence and uniqueness of the solution of a non steady free boundary problem, Boll. Un. Mat. Ital. B (5) 14 (1977), 423-466.

[12] E. Zaouche, Uniqueness of solution in a rectangular domain of an evolution dam problem with heterogeneous coefficients, Electron. J. Differ. Equations 2018, No. 169, 17 pp.

E. Zaouche

Department of Mathematics

University of EL Oued

B. P. 789 El Oued 39000

Labo. Part. Diff. Eq. \& Hist. Maths

Ecole Normale Supérieure

16050 Vieux-Kouba Algiers

Algeria

E-mail: elmehdi-zaouche@univ-eloued.dz

Received: 4.2.2019.

Revised: 10.9.2019. 